International Journal of Pure and Applied Mathematics

Volume 103 No. 3 2015, 453-466

ISSN: 1311-8080 (printed version); ISSN: 1314-3395 (on-line version)

url: http://www.ijpam.eu

doi: http://dx.doi.org/10.12732/ijpam.v103i3.7

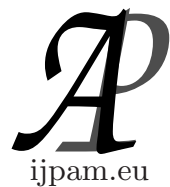

\title{
INTUITIONISTIC FUZZY CO-TAUTOLOGICAL MATRIX
}

\author{
P. Murugadas ${ }^{1 \S}$, K. Lalitha ${ }^{2}$ \\ 1,2 Mathematics Section \\ Faculty of Engineering and Technology \\ Annamalai University, Annamalainagar \\ Tamil Nadu, INDIA
}

\begin{abstract}
In this paper, we study some properties of intuitionistic fuzzy co-tautological matrix using intuitionistic fuzzy implication operator.
\end{abstract}

AMS Subject Classification: 03E72, 15B15

Key Words: intuitionistic fuzzy matrix (IFM), intuitionistic fuzzy set (IFS), intuitionistic fuzzy implication operator (IFIO), intuitionistic fuzzy tautological matrix (IFTM), intuitionistic fuzzy co-tautological matrix (IFCTM)

\section{Introduction}

Since Zadeh's [24] introduction of the compositional rule of inference many researchers have used fuzzy implication operator to represent the relation between two variables linked together by means of an if-then rule. A very important part of research in fuzzy logic focuses on extending the classical binary logic operators negation $(\neg)$, conjunction $(\wedge)$, disjunction $(\vee)$ and implication $(\rightarrow)$ to fuzzy logic operators.

The concept of intuitionistic fuzzy propositional calculus was introduced by Atanassov [2] before three decades and after that in a series of papers a lot of new implications are defined over IFSs [3]-[7].

All the above implication operators are defined for IFS only. After the

Received: April 25, 2015

(c) 2015 Academic Publications, Ltd.

$\S$ Correspondence author url: www.acadpubl.eu 
introduction of IFM by Im et al. [13], the intuitionistic fuzzy implication operators has been used in IFM by Sriram and Murugadas [20] and Murugadas and Lalitha [16]-[19] which was the extension of Godel implication used by Hiroshi hasimoto [12] in fuzzy matrix theory. The Godel implication $y \rightarrow x($ Fig.1) gives value $x$ if $x<y$ (1 if $x \geq y$ ) for all $x, y \in[0,1]$. Hiroshi hasimoto [11] used another implication $y \rightarrow x($ Fig.2) which takes values 0 if $x \leq y(x$ if $x>y)$ and studied the relation between $\rightarrow$ and $\rightarrow$.

$I_{G}$ (Godel Implication $\rightarrow$ ) is false when the antecedent is true and the corresponding consequent is false, but the implication $\rightarrow$ is true only when the consequent is true with false antecedent; in all other cases it is false.
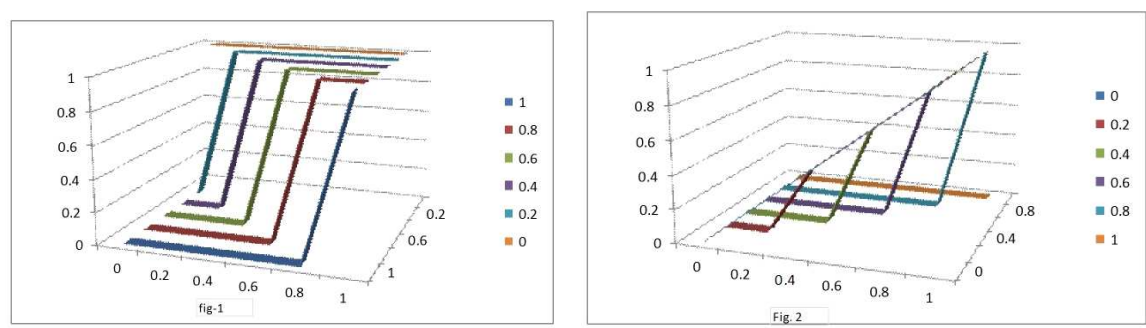

Figure 1

An interesting extension of Fuzzy Set theory is intuitionistic fuzzy set theory by Atanassov [2]; Atanassov interpret the Zadeh's fuzzy set as, if $X$ is a non empty set, an intuitionistic fuzzy set (IFS) $A$ in $X$ (universal set) is defined as an object of the following form $A=\left\{\left\langle x, \mu_{A}(x), \nu_{A}(x)\right\rangle / x \in X\right\}$, where the functions: $\mu_{A}(x): X \rightarrow[0,1]$ and $\nu_{A}(x): X \rightarrow[0,1]$ define the membership function and non-membership function of the element $x \in X$ respectively and for every $x \in X: 0 \leq \mu_{A(x)}+\nu_{A}(x) \leq 1$. Xu, Yager [23] represents $\left\langle\mu_{A}(x), \nu_{A}(x)\right\rangle$ as intuitionistic fuzzy values with $\mu_{A(x)}+\nu_{A}(x) \leq 1$.

An IFS $A$ is an intuitionistic fuzzy tautological set (Intuitionistic fuzzy cotautological sets) [3] if and only if for every $x \in X, \mu_{A(x)} \geq \nu_{A}(x)\left(\mu_{A}(x) \leq\right.$ $\left.\nu_{A}(x)\right)$ holds, and a tautological set (co-tautological set) if and only if for every $x \in X, \mu_{A}(x)=1, \nu_{A}(x)=0\left(\mu_{A}(x)=0, \nu_{A}(x)=1\right)$ holds. For simplicity, we consider the pair $\left\langle x, x^{\prime}\right\rangle$ as membership and non-member function of an IFS with $x+x^{\prime} \leq 1$. Also, we can interpret an element in IFS in a classical way as $(1,0)$, one being the membership degree and zero being the non membership degree; and an element does not belong to the IFS when the membership degree is zero and the non-membership degree is one, $(0,1)$.

A fuzzy implication $\mathrm{I}$, is a function of the form $I:[0,1]^{2} \longrightarrow[0,1]$, which for any possible truth values $x, y$ of the given fuzzy proposition $p, q$ respectively, 
defines the truth value, $I(x, y)$ of the conditional proposition "if $p$ then $q$ ". This function should be an extension of the classical implication $p \rightarrow q$, from the restricted domain $(0,1)$ to the full domain $[0,1]$ of truth values in fuzzy logic. This can be extended in an intuitionistic fuzzy sense, when the propositions $p, q$ of the conditional if $p$ then $q$ are intuitionistic fuzzy. That is, when each one of them is defined by two values, where the first indicates the degree of truth, of the proposition and the second the degree of non-truth. The intuitionistic fuzzy truth value is $\langle 1,0\rangle$ and non-truth value is $\langle 0,1\rangle$. This implication operator must be an extension of fuzzy implication in the sense of J. Fodor and M. Roubens [10]. Atanassov and Gargov [7] and later Conelis and Deschrijver [8, 9] gave the following definition of intuitionistic fuzzy implication operator.

An intuitionistic fuzzy implication is any $I_{I}: D^{2} \longrightarrow D$ mapping satisfying the border conditions:

$$
\begin{aligned}
I_{I}((0,1),(0,1)) & =(1,0), \\
I_{I}((0,1),(1,0))=(1,0), I_{I}((1,0),(1,0)) & =(1,0), I_{I}((1,0),(0,1))=(0,1),
\end{aligned}
$$

and the two following conditions:

1. If $(x, y) \leq\left(x^{\prime}, y^{\prime}\right)$ then $I_{I}((x, y),(z, t)) \geq I_{I}\left(\left(x^{\prime}, y^{\prime}\right),(z, t)\right)$ for all $(z, t) \in$ $D$;

2. If $(z, t) \leq\left(z^{\prime}, t^{\prime}\right)$ then $I_{I}((x, y),(z, t)) \leq I_{I}\left(\left(x^{\prime}, y^{\prime}\right),\left(z^{\prime}, t^{\prime}\right)\right)$ for all $(z, t) \in$ $D$.

Meenakhsi and Gandhimathi [15], Sriram and Murugadas [20]-[22] and Khan, Mathumangal Pal and Shyamal [14] developed intuitionistic fuzzy matrix theory in finding the $g$ - inverse, intuitionistic fuzzy linear equation, intuitionistic fuzzy linear transformation etc. Sriram and Murugadas [20] extended the implication operator $\rightarrow$ to IFM and discussed several properties like subinverse, semi-inverse and necessary and sufficient condition for the existence of $g$-inverse using the implication operator. The authors in [16] introduced hook implication operator $\hookleftarrow$ for IFS as well as IFM, discussed the relation with $\rightarrow$ implication operator and obtained maximum solution (minimum solution) for the inequality $A \times X \times B \leqslant C,(A \diamond X \diamond B \geqslant C)$ using max-min (min-max) product.

The authors in [17] defined bi-implication operator for IFS, extended it to IFM, its relation with IFIO and obtained sub-inverses and $g$-inverses of an IFM. The authors in [19] used $\rightarrow$ implication operator in IFM and proved some results for IFTMs. On this paper, we use $\rightarrow$ operator in IFM and study some properties of Co-tautological matrix. 


\section{Preliminaries}

Definition 1. [21] For $\left\langle x, x^{\prime}\right\rangle,\left\langle y, y^{\prime}\right\rangle \in I F S$, define:

$$
\begin{aligned}
& \left\langle x, x^{\prime}\right\rangle \vee\left\langle y, y^{\prime}\right\rangle=\left\langle\max \{x, y\}, \min \left\{x^{\prime}, y^{\prime}\right\}\right\rangle ; \\
& \left\langle x, x^{\prime}\right\rangle \wedge\left\langle y, y^{\prime}\right\rangle=\left\langle\min \{x, y\}, \max \left\{x^{\prime}, y^{\prime}\right\}\right\rangle ; \\
& \left\langle x, x^{\prime}\right\rangle^{c}=\left\langle x^{\prime}, x\right\rangle . \text { (complement). }
\end{aligned}
$$

Definition 2. [21] Let $X=\left\{x_{1}, x_{2}, \ldots x_{m}\right\}$ be a set of alternatives and $Y=\left\{y_{1}, y_{2}, \ldots y_{n}\right\}$ be the attribute set of each element of $X$. An intuitionistic fuzzy matrix (IFM) is defined by $A=\left(\left\langle\left(x_{i}, y_{j}\right), \mu_{A}\left(x_{i}, y_{j}\right), \nu_{A}\left(x_{i}, y_{j}\right)\right\rangle\right)$ for $i=$ $1,2 \cdots m$ and $j=1,2, \cdots n$, where $\mu_{A}: X \times Y \rightarrow[0,1]$ and $\nu_{A}: X \times Y \rightarrow[0,1]$ satisfy the condition $0 \leq \mu_{A}\left(x_{i}, y_{j}\right)+\nu_{A}\left(x_{i}, y_{j}\right) \leq 1$. For simplicity we denote an intuitionistic fuzzy matrix (IFM) is a matrix of pairs $A=\left(\left\langle a_{i j}, a_{i j}^{\prime}\right\rangle\right)$ of non negative real numbers satisfying $a_{i j}+a_{i j}^{\prime} \leq 1$ for all $i, j$. We denote the set of all IFM of order $m \times n$ by $\mathscr{F}_{m n}$.

For any two elements $A=\left(\left\langle a_{i j}, a_{i j}^{\prime}\right\rangle\right), B=\left(\left\langle b_{i j}, b_{i j}^{\prime}\right\rangle\right) \in \mathscr{F}_{m n}$, define

1. $A \vee B=\left(\left\langle a_{i j} \vee b_{i j}, a_{i j}^{\prime} \wedge b_{i j}^{\prime}\right\rangle\right)=A \oplus B$, (component wise addition)

2. $A \wedge B=\left(\left\langle a_{i j} \wedge b_{i j}, a_{i j}^{\prime} \vee b_{i j}^{\prime}\right\rangle\right)=A \odot B$, (component wise multiplication) for all $1 \leq i \leq m$ and $1 \leq j \leq n$.

3. $J=(\langle 1,0\rangle)$ the Universal matrix(matrix in which all entries are $\langle 1,0\rangle)$

4. $I=\left(\left\langle\delta_{i j}, \delta_{i j}^{\prime}\right\rangle\right)\left(\right.$ Identity Matrix) where $\left\langle\delta_{i j}, \delta_{i j}^{\prime}\right\rangle= \begin{cases}\langle 1,0\rangle & \text { if } i=j \\ \langle 0,1\rangle & \text { if } i \neq j\end{cases}$

5. $A \geq B$ if $a_{i j} \geq b_{i j}$ and $a_{i j}^{\prime} \leq b_{i j}^{\prime}$ for all $i, j$, and $A>B$ if $a_{i j}>b_{i j}$ and $a_{i j}^{\prime}<b_{i j}^{\prime}$ for all $i, j$. (In which case $A$ and $B$ are comparable.)

6. $\bar{A}=\left(\left\langle a_{i j}^{\prime}, a_{i j}\right\rangle\right)($ complement of $A)$.

Amar Kumar et. al., [1] proved that generalized IFM forms a distributive lattice using this component wise addition $\oplus$ and component wise multiplication $\odot$.

Definition 3. [16] For $\left\langle x, x^{\prime}\right\rangle,\left\langle y, y^{\prime}\right\rangle \in$ IFS define $\left\langle y, y^{\prime}\right\rangle \rightarrow\left\langle x, x^{\prime}\right\rangle= \begin{cases}\left\langle x, x^{\prime}\right\rangle & \text { if }\left\langle x, x^{\prime}\right\rangle>\left\langle y, y^{\prime}\right\rangle \\ \langle 0,1\rangle & \text { if }\left\langle x, x^{\prime}\right\rangle \leq\left\langle y, y^{\prime}\right\rangle\end{cases}$ 
$\left\langle y, y^{\prime}\right\rangle \rightarrow\left\langle x, x^{\prime}\right\rangle= \begin{cases}\langle 1,0\rangle & \text { if }\left\langle x, x^{\prime}\right\rangle \geq\left\langle y, y^{\prime}\right\rangle \\ \left\langle x, x^{\prime}\right\rangle & \text { if }\left\langle x, x^{\prime}\right\rangle<\left\langle y, y^{\prime}\right\rangle\end{cases}$

It is easy to verify that IFIO $\rightarrow$ satisfies border conditions and condition 1 and 2 of the definition of IFIO defined by the authors [7, 8, 9, 10], but the IFIO $\rightarrow$ satisfies condition 1 and 2 and has different border conditions.

\section{Properties of IFCTMs}

Throughout this section matrices means IFMs and they are comparable.

Definition 4. $A \rightarrow B=\left(\left\langle a_{i j}, a_{i j}^{\prime}\right\rangle \rightarrow\left\langle b_{i j}, b_{i j}^{\prime}\right\rangle\right)$. (Here $\rightarrow$ is used component wise.)

Definition 5. An intuitionistic fuzzy matrix is called an intuitionistic fuzzy tautological matrix (IFTM) if and only if $a_{i j} \geq a_{i j}^{\prime}$ for all $i, j$.

Definition 6. An intuitionistic fuzzy matrix is called an intuitionistic fuzzy co-tautological matrix (IFCTM) if and only if $a_{i j} \leq a_{i j}^{\prime}$ for all $i, j$.

Property 7. Let $A$ and $B$ be two IFMs then the following expressions are IFCTMs.

1. $A \rightarrow A$.

2. $A \rightarrow \bar{A}$ (if $A$ is a tautological matrix).

3. $A \neg(B \neg A)$.

Proof.

1. Let $A=\left\langle a_{i j}, a_{i j}^{\prime}\right\rangle$

As $\left\langle a_{i j}, a_{i j}^{\prime}\right\rangle=\left\langle a_{i j}, a_{i j}^{\prime}\right\rangle$,

then $\left\langle a_{i j}, a_{i j}\right\rangle \rightarrow\left\langle a_{i j}, a_{i j}^{\prime}\right\rangle=\langle 0,1\rangle$ for all $i, j$.

So, $A \rightarrow A$ is an IFCTMs.

2. $A=\left\langle a_{i j}, a_{i j}^{\prime}\right\rangle$

$\bar{A}=\left\langle a_{i j}^{\prime}, a_{i j}\right\rangle$ 
If $A$ is IFTM then $a_{i j} \geq a_{i j}^{\prime}$ for all $i, j$

Then $A \rightarrow \bar{A}=(\langle 0,1\rangle)\left(\right.$ since $\left\langle a_{i j}^{\prime}, a_{i j}\right\rangle \leq\left\langle a_{i j}, a_{i j}^{\prime}\right\rangle$ for all $i, j$.)

3. Let $A=\left(\left\langle a_{i j}, a_{i j}^{\prime}\right\rangle\right)$

$B=\left(\left\langle b_{i j}, b_{i j}^{\prime}\right\rangle\right)$

$B \rightarrow A=\left(\left\langle b_{i j}, b_{i j}^{\prime}\right\rangle\right) \rightarrow\left(\left\langle a_{i j}, a_{i j}^{\prime}\right\rangle\right)$

Case (i): If $\left\langle b_{i j}, b_{i j}^{\prime}\right\rangle<\left\langle a_{i j}, a_{i j}^{\prime}\right\rangle$

then $\left\langle b_{i j}, b_{i j}^{\prime}\right\rangle \rightarrow\left\langle a_{i j}, a_{i j}^{\prime}\right\rangle=\left\langle a_{i j}, a_{i j}^{\prime}\right\rangle$

$B \rightarrow A=\left(\left\langle a_{i j}, a_{i j}\right\rangle\right)$

$A \rightarrow(B \rightarrow A)=\left(\left\langle a_{i j}, a_{i j}^{\prime}\right\rangle\right) \rightarrow\left(\left\langle a_{i j}, a_{i j}^{\prime}\right\rangle\right)$

$A \rightarrow(B \rightarrow A)=(\langle 0,1\rangle)$

Case (ii): If $\left\langle b_{i j}, b_{i j}^{\prime}\right\rangle \geq\left\langle a_{i j}, a_{i j}^{\prime}\right\rangle$

$B \rightarrow A=\left(\left\langle b_{i j}, b_{i j}\right\rangle\right) \rightarrow\left(\left\langle a_{i j}, a_{i j}^{\prime}\right\rangle\right)=(\langle 0,1\rangle)$

$A \rightarrow(B \rightarrow A)=\left(\left\langle a_{i j}, a_{i j}^{\prime}\right\rangle\right) \rightarrow(\langle 0,1\rangle)$

$A \rightarrow(B \rightarrow A)=(\langle 0,1\rangle)$

$\therefore A \rightarrow(B \rightarrow A)$ is an IFCTMs.

Property 8. Let $A$ and $B$ be two IFMs then

1. $(A \vee B) \rightarrow A$

2. $(A \vee B) \rightarrow B$

3. $A \neg(B \neg(A \wedge B))$ are IFCTMs.

Proof.

1. $A \vee B=\left(\left\langle a_{i j}, a_{i j}^{\prime}\right\rangle \vee\left\langle b_{i j}, b_{i j}^{\prime}\right\rangle\right)$

Case (i): If $\left\langle a_{i j}, a_{i j}^{\prime}\right\rangle \geq\left\langle b_{i j}, b_{i j}^{\prime}\right\rangle$

$(A \vee B) \rightarrow A=\left(\left\langle a_{i j}, a_{i j}^{\prime}\right\rangle\right) \rightarrow\left(\left\langle a_{i j}, a_{i j}^{\prime}\right\rangle\right)$

$(A \vee B) \rightarrow A=(\langle 0,1\rangle)$

Case (ii): If $\left\langle a_{i j}, a_{i j}^{\prime}\right\rangle<\left\langle b_{i j}, b_{i j}^{\prime}\right\rangle$,

$(A \vee B) \rightarrow A=\left\langle b_{i j}, b_{i j}^{\prime}\right\rangle \rightarrow\left\langle a_{i j}, a_{i j}^{\prime}\right\rangle$

$(A \vee B) \rightarrow A=(\langle 0,1\rangle)$

2. Case (i): $\left\langle a_{i j}, a_{i j}^{\prime}\right\rangle \geq\left\langle b_{i j}, b_{i j}^{\prime}\right\rangle$

$(A \vee B) \rightarrow B=\left(\left\langle a_{i j}, a_{i j}^{\prime}\right\rangle\right) \rightarrow\left(\left\langle b_{i j}, b_{i j}^{\prime}\right\rangle\right)$

$=(\langle 0,1\rangle)$

Case (ii): $\left\langle a_{i j}, a_{i j}^{\prime}\right\rangle\left\langle\left\langle b_{i j}, b_{i j}^{\prime}\right\rangle\right.$ 
$(A \vee B) \rightarrow B=\left\langle b_{i j}, b_{i j}^{\prime}\right\rangle \rightarrow\left\langle b_{i j}, b_{i j}^{\prime}\right\rangle$

$=(\langle 0,1\rangle)$

3. Case (i): $A \wedge B=\left(\left\langle a_{i j}, a_{i j}^{\prime}\right\rangle \wedge\left\langle b_{i j}, b_{i j}^{\prime}\right\rangle\right)$

If $\left\langle a_{i j}, a_{i j}^{\prime}\right\rangle \geq\left\langle b_{i j}, b_{i j}^{\prime}\right\rangle$

$A \wedge B=\left(\left\langle b_{i j}, b_{i j}^{\prime}\right\rangle\right)$

$(B \rightarrow(A \wedge B))=\left(\left\langle b_{i j}, b_{i j}^{\prime}\right\rangle\right) \rightarrow\left(\left\langle b_{i j}, b_{i j}^{\prime}\right\rangle\right)$

$=(\langle 0,1\rangle)$

$A \neg(B \neg(A \wedge B))=\left(\left\langle a_{i j}, a_{i j}^{\prime}\right\rangle\right) \rightarrow(\langle 0,1\rangle)$

$=(\langle 0,1\rangle)$

Case (ii): If $\left\langle a_{i j}, a_{i j}^{\prime}\right\rangle\left\langle\left\langle b_{i j}, b_{i j}^{\prime}\right\rangle\right.$

$A \wedge B=\left(\left\langle a_{i j}, a_{i j}^{\prime}\right\rangle\right)$

$(B \rightarrow(A \wedge B))=\left(\left\langle b_{i j}, b_{i j}^{\prime}\right\rangle\right) \rightarrow\left(\left\langle a_{i j}, a_{i j}^{\prime}\right\rangle\right)$

$=(\langle 0,1\rangle)$

$A \neg(B \rightarrow(A \wedge B))=\left(\left\langle a_{i j}, a_{i j}^{\prime}\right\rangle\right) \rightarrow(\langle 0,1\rangle)$

$=(\langle 0,1\rangle)$

So, $A \neg(B \neg(A \wedge B))$ is an IFCTM.

Property 9. If $A$ and $B$ are IFMs then

1. $A \rightarrow(A \wedge B)$

2. $B \rightarrow(A \wedge B)$ are IFCTMs

Proof.

1. Case (i): $A \wedge B=\left(\left\langle a_{i j}, a_{i j}^{\prime}\right\rangle \wedge\left\langle b_{i j}, b_{i j}^{\prime}\right\rangle\right)$

If $\left\langle a_{i j}, a_{i j}^{\prime}\right\rangle \leq\left\langle b_{i j}, b_{i j}^{\prime}\right\rangle$

$A \wedge B=\left\langle a_{i j}, a_{i j}^{\prime}\right\rangle$

$A \rightarrow A \wedge B=\left(\left\langle a_{i j}, a_{i j}^{\prime}\right\rangle\right) \rightarrow\left(\left\langle a_{i j}, a_{i j}^{\prime}\right\rangle\right)$

$=(\langle 0,1\rangle)$

Case (ii): If $\left(\left\langle a_{i j}, a_{i j}^{\prime}\right\rangle\right)>\left(\left\langle b_{i j}, b_{i j}^{\prime}\right\rangle\right)$

$A \wedge B=\left(\left\langle b_{i j}, b_{i j}^{\prime}\right\rangle\right)$

$A \rightarrow A \wedge B=\left(\left\langle a_{i j}, a_{i j}^{\prime}\right\rangle\right) \rightarrow\left(\left\langle b_{i j}, b_{i j}^{\prime}\right\rangle\right)$

$=(\langle 0,1\rangle)$

Hence $A \rightarrow(A \wedge B)$ is an IFCTM.

2. Case (i): If $\left\langle a_{i j}, a_{i j}^{\prime}\right\rangle \leq\left\langle b_{i j}, b_{i j}^{\prime}\right\rangle$

$A \wedge B=\left(\left\langle a_{i j}, a_{i j}\right\rangle\right)$ 
$B \rightarrow(A \wedge B)=\left(\left\langle b_{i j}, b_{i j}^{\prime}\right\rangle\right) \rightarrow\left(\left\langle a_{i j}, a_{i j}^{\prime}\right\rangle\right)$

$=(\langle 0,1\rangle)$

Case (ii): If $\left\langle a_{i j}, a_{i j}^{\prime}\right\rangle>\left\langle b_{i j}, b_{i j}^{\prime}\right\rangle$

$A \wedge B=\left(\left\langle b_{i j}, b_{i j}^{\prime}\right\rangle\right)$

$B \rightarrow(A \wedge B)=\left(\left\langle b_{i j}, b_{i j}^{\prime}\right\rangle\right) \rightarrow\left(\left\langle b_{i j}, b_{i j}^{\prime}\right\rangle\right)$

$=(\langle 0,1\rangle)$

Hence $B \rightarrow(A \wedge B)$ is an IFCTM.

Property 10. For any three IFMS A, B, C the following expressions are IFCTMs.

1. $(A \rightarrow C) \rightarrow((B \rightarrow C) \rightarrow((A \vee B) \rightarrow C))$

2. $((A \rightarrow B) \rightarrow(A \rightarrow C)) \rightarrow(A \rightarrow(B \rightarrow C))$

3. $((A \neg \bar{B}) \rightarrow B) \rightarrow(A \rightarrow \bar{B})$ (if $B$ is an IFTM)

\section{Proof.}

1. Case (i) $A \leq B$

Sub case(i) $A \leq B<C$

$A \rightarrow C=\left(\left\langle c_{i j}, c_{i j}^{\prime}\right\rangle\right)$

$B \rightarrow C=\left(\left\langle c_{i j}, c_{i j}\right\rangle\right)$

$A \vee B=\left(\left\langle b_{i j}, b_{i j}^{\prime}\right\rangle\right)$

$(A \vee B) \rightarrow C=\left(\left\langle b_{i j}, b_{i j}^{\prime}\right\rangle\right) \rightarrow\left(\left\langle c_{i j}, c_{i j}^{\prime}\right\rangle\right)$

$(A \vee B) \rightarrow C=\left(\left\langle c_{i j}, c_{i j}\right\rangle\right)$

$((B \rightarrow C) \rightarrow((A \vee B) \rightarrow C))=\left\langle c_{i j}, c_{i j}^{\prime}\right\rangle \rightarrow\left\langle c_{i j}, c_{i j}^{\prime}\right\rangle$

$=(\langle 0,1\rangle)$

$(A \rightarrow C) \rightarrow((B \rightarrow C) \rightarrow((A \vee B) \rightarrow C))$

$=\left(\left\langle c_{i j}, c_{i j}\right\rangle\right) \rightarrow(\langle 0,1\rangle)$

$=(\langle 0,1\rangle)$

Sub case(ii) If $C<A \leq B$

$A \rightarrow C=(\langle 0,1\rangle)$

$B \rightarrow C=(\langle 0,1\rangle)$

$A \vee B=\left(\left\langle b_{i j}, b_{i j}^{\prime}\right\rangle\right)$

$(A \vee B) \rightarrow C=\left(\left\langle b_{i j}, b_{i j}^{\prime}\right\rangle\right) \rightarrow\left(\left\langle c_{i j}, c_{i j}^{\prime}\right\rangle\right)$

$(A \vee B) \rightarrow C=(\langle 0,1\rangle)$

$((B \rightarrow C) \rightarrow((A \vee B) \rightarrow C))=(\langle 0,1\rangle) \rightarrow(\langle 0,1\rangle)$

$=(\langle 0,1\rangle)$ 


$$
\begin{aligned}
& (A \neg C) \rightarrow((B \neg C) \rightarrow((A \vee B) \rightarrow C)) \\
& =(\langle 0,1\rangle) \rightarrow(\langle 0,1\rangle) \\
& =(\langle 0,1\rangle)
\end{aligned}
$$

Sub case(iii) If $A \leq C \leq B$

$$
\begin{aligned}
& A \rightarrow C=\left(\left\langle a_{i j}, a_{i j}^{\prime}\right\rangle\right) \rightarrow\left(\left\langle c_{i j}, c_{i j}^{\prime}\right\rangle\right) \\
& = \begin{cases}(\langle 0,1\rangle) & \text { if }\left\langle a_{i j}, a_{i j}^{\prime}\right\rangle=\left\langle c_{i j}, c_{i j}^{\prime}\right\rangle \text { for all } i, j \\
\left(\left\langle c_{i j}, c_{i j}^{\prime}\right\rangle\right) & \text { if }\left\langle a_{i j}, a_{i j}^{\prime}\right\rangle<\left\langle c_{i j}, c_{i j}^{\prime}\right\rangle\end{cases} \\
& B \rightarrow C=\left(\left\langle b_{i j}, b_{i j}^{\prime}\right\rangle\right) \rightarrow\left(\left\langle c_{i j}, c_{i j}^{\prime}\right\rangle\right) \\
& =(\langle 0,1\rangle) \\
& (A \vee B) \rightarrow C=\left(\left\langle b_{i j}, b_{i j}^{\prime}\right\rangle\right) \rightarrow\left(\left\langle c_{i j}, c_{i j}^{\prime}\right\rangle\right) \\
& =\left(\left\langle b_{i j}, b_{i j}^{\prime}\right\rangle\right) \rightarrow\left(\left\langle c_{i j}, c_{i j}\right\rangle\right) \\
& =(\langle 0,1\rangle) \\
& ((B \neg C) \rightarrow((A \vee B) \rightarrow C))=(\langle 0,1\rangle) \rightarrow(\langle 0,1\rangle) \\
& =(\langle 0,1\rangle) \\
& \therefore(A \neg C) \rightarrow((B \neg C) \rightarrow((A \vee B) \rightarrow C)) \\
& =(A \neg C) \rightarrow(\langle 0,1\rangle) \\
& =(\langle 0,1\rangle) \text { whatever be the value of }(A \rightarrow C)) .
\end{aligned}
$$

2. Case (i) $A \leq B$

Sub case(i) $A \leq B<C$

$A \rightarrow C=\left(\left\langle c_{i j}, c_{i j}^{\prime}\right\rangle\right)$

$B \rightarrow C=\left(\left\langle c_{i j}, c_{i j}\right\rangle\right)$

$A \rightarrow B= \begin{cases}(\langle 0,1\rangle) & \text { if }\left\langle a_{i j}, a_{i j}^{\prime}\right\rangle=\left\langle b_{i j}, b_{i j}^{\prime}\right\rangle \text { for all } i, j \\ \left(\left\langle b_{i j}, b_{i j}^{\prime}\right\rangle\right) & \text { if }\left\langle a_{i j}, a_{i j}^{\prime}\right\rangle<\left\langle b_{i j}, b_{i j}^{\prime}\right\rangle\end{cases}$

$((A \neg B) \neg(A \neg C)) \rightarrow(A \neg(B \neg C))=((A \neg B) \neg(A \neg C)) \rightarrow$ $\left.(A \rightarrow C))=\left((A \rightarrow B) \rightarrow\left(\left\langle c_{i j}, c_{i j}^{\prime}\right\rangle\right)\right) \rightarrow\left(\left\langle c_{i j}, c_{i j}^{\prime}\right\rangle\right)=\left(\left\langle c_{i j}, c_{i j}^{\prime}\right\rangle\right)\right) \rightarrow$ $\left(\left\langle c_{i j}, c_{i j}^{\prime}\right\rangle\right)=(\langle 0,1\rangle)($ Since $A \rightarrow B<C)$

Sub case(ii) If $C<A \leq B$

$A \rightarrow C=(\langle 0,1\rangle)$

$B \rightarrow C=(\langle 0,1\rangle)$

$((A \neg B) \rightarrow(A \neg C)) \rightarrow(A \neg(B \neg C))=((A \neg B) \rightarrow(\langle 0,1\rangle)) \rightarrow$ $(A \neg(\langle 0,1\rangle))=((A \neg B) \neg(\langle 0,1\rangle)) \rightarrow(\langle 0,1\rangle)=(\langle 0,1\rangle) \rightarrow(\langle 0,1\rangle)=$ $(\langle 0,1\rangle)$.

Sub case (iii) If $A \leq C \leq B$

$$
\begin{aligned}
& A \rightarrow C=\left(\left\langle a_{i j}, a_{i j}^{\prime}\right\rangle\right) \rightarrow\left(\left\langle c_{i j}, c_{i j}^{\prime}\right\rangle\right) \\
& = \begin{cases}(\langle 0,1\rangle) & \text { if }\left\langle a_{i j}, a_{i j}^{\prime}\right\rangle=\left\langle c_{k j}, c_{k j}^{\prime}\right\rangle \text { for all } i, j \\
\left(\left\langle c_{i j}, c_{i j}^{\prime}\right\rangle\right) & \text { if }\left\langle a_{i j}, a_{i j}^{\prime}\right\rangle<\left\langle c_{i j}, c_{i j}^{\prime}\right\rangle\end{cases} \\
& B \rightarrow C=\left(\left\langle b_{i j}, b_{i j}^{\prime}\right\rangle\right) \rightarrow\left(\left\langle c_{i j}, c_{i j}\right\rangle\right)
\end{aligned}
$$




$$
\begin{aligned}
& =(\langle 0,1\rangle) \\
& A \rightarrow B= \begin{cases}(\langle 0,1\rangle) & \text { if }\left\langle a_{i j}, a_{i j}^{\prime}\right\rangle=\left\langle b_{i j}, b_{i j}^{\prime}\right\rangle \text { for all } i, j \\
\left(\left\langle b_{i j}, b_{i j}^{\prime}\right\rangle\right) & \text { if }\left\langle a_{i j}, a_{i j}^{\prime}\right\rangle<\left\langle b_{i j}, b_{i j}^{\prime}\right\rangle\end{cases} \\
& ((A \rightarrow B) \rightarrow(A \rightarrow C)) \rightarrow(A \rightarrow(B \rightarrow C)) \\
& =((A \rightarrow B) \rightarrow(A \rightarrow C)) \rightarrow(A \rightarrow(\langle 0,1\rangle))=((A \rightarrow B) \rightarrow(A \rightarrow C)) \rightarrow \\
& (\langle 0,1\rangle)=(\langle 0,1\rangle)(\text { whatever be the value of }(A \rightarrow B) \rightarrow(A \rightarrow C)) .
\end{aligned}
$$

3. Case (i). If $A \geq B \geq \bar{B}$ (Since $B$ is an IFTM $B \geq \bar{B}$ )

$((A \neg \bar{B}) \rightarrow B) \rightarrow(A \rightarrow \bar{B})=((\langle 0,1\rangle) \rightarrow B) \rightarrow(\langle 0,1\rangle)=(\langle 0,1\rangle)$ for any value of $(\langle 0,1\rangle) \rightarrow B$.

Case (ii). $A<B$

Sub case (i) $A<\bar{B}<B$

$((A \neg \bar{B}) \longrightarrow B) \longrightarrow(A \neg \bar{B})=(\bar{B} \neg B) \neg \bar{B}=B \neg \bar{B}=(\langle 0,1\rangle)$.

Sub case (ii) $\bar{B}<A<B$

$(A \neg \bar{B})=(\langle 0,1\rangle)$ so $((A \rightarrow \bar{B}) \rightarrow B) \rightarrow(A \rightarrow \bar{B})=(\langle 0,1\rangle)$ for any value of $(A \rightarrow \bar{B}) \rightarrow B$.

Property 11. If $A>B$ then $A \rightarrow B$ is an IFCTM.

Proof. Given $A>B$, then $A \rightarrow B=(\langle 0,1\rangle)$

So, $A \rightarrow B$ is a IFCTM.

Property 12. If $A, B$ are IFMs then the following expressions are IFCTMs.

1. $B \rightarrow(A \wedge(A \rightarrow B))$

2. $((B \neg A) \vee B) \rightarrow A$

3. $((A \neg B) \vee A) \rightarrow B$

4. $((A \rightarrow B) \vee(B \rightarrow C)) \rightarrow(A \rightarrow C)$

5. $B \rightarrow((A \vee B) \rightarrow B)$

6. $((B \neg C) \rightarrow((A \wedge B) \rightarrow C)) \rightarrow(A \neg C)$

Proof. 1. Case (i): If $A<B$

$$
\begin{aligned}
& A \neg B=B \\
& B \neg(A \wedge(A \neg B))=B \neg(A \wedge B)=B \neg A=(\langle 0,1\rangle)
\end{aligned}
$$


Case (ii): If $A \geq B$

$A \rightarrow B=(\langle 0,1\rangle)$

$B \neg(A \wedge(A \neg B))=B \neg(A \wedge(\langle 0,1\rangle))=B \neg(\langle 0,1\rangle)=(\langle 0,1\rangle)$

2. Case (i): If $A<B$

$B \rightarrow A=(\langle 0,1\rangle)$

$((B \neg A) \vee B) \rightarrow A=((\langle 0,1\rangle) \vee B) \neg A=B \neg A=(\langle 0,1\rangle)$

Case (ii): If $A \geq B$

$B \rightarrow A=A$

$((B \neg A) \vee B) \rightarrow A=(A \vee B) \rightarrow A=A \neg A=(\langle 0,1\rangle)$

3. Case (i): If $A \geq B$

$A \rightarrow B=(\langle 0,1\rangle)$

$((A \neg B) \vee A) \rightarrow B=A \neg B=(\langle 0,1\rangle)$

Case (ii): If $A<B$

$A \rightarrow B=B$

$((A \neg B) \vee A) \neg B=B \neg B=(\langle 0,1\rangle)$

4. Case (i): $A \leq B<C$

$A \rightarrow C=C, B \rightarrow C=C$,

$A \rightarrow B= \begin{cases}(\langle 0,1\rangle) & \text { if }\left\langle a_{i j}, a_{i j}^{\prime}\right\rangle=\left\langle b_{i j}, b_{i j}^{\prime}\right\rangle \text { for all } i, j \\ \left(\left\langle b_{i j}, b_{i j}^{\prime}\right\rangle\right) & \text { if }\left\langle a_{i j}, a_{i j}^{\prime}\right\rangle<\left\langle b_{i j}, b_{i j}^{\prime}\right\rangle\end{cases}$

$((A \neg B) \vee(B \neg C)) \rightarrow(A \neg C)=((A \neg B) \vee C)) \rightarrow C=C \rightarrow C=$ $(\langle 0,1\rangle)$

Case (ii): If $A \leq C \leq B$

$B \rightarrow C=(\langle 0,1\rangle)$,

$A \rightarrow B= \begin{cases}(\langle 0,1\rangle) & \text { if }\left\langle a_{i j}, a_{i j}^{\prime}\right\rangle=\left\langle b_{i j}, b_{i j}^{\prime}\right\rangle \text { for all } i, j \\ \left(\left\langle b_{i j}, b_{i j}^{\prime}\right\rangle\right) & \text { if }\left\langle a_{i j}, a_{i j}^{\prime}\right\rangle<\left\langle b_{i j}, b_{i j}^{\prime}\right\rangle\end{cases}$

$A \rightarrow C=\left(\left\langle a_{i j}, a_{i j}^{\prime}\right\rangle\right) \rightarrow\left(\left\langle c_{i j}, c_{i j}^{\prime}\right\rangle\right)$

$= \begin{cases}(\langle 0,1\rangle) & \text { if }\left\langle a_{i j}, a_{i j}^{\prime}\right\rangle=\left\langle c_{k j}, c_{k j}^{\prime}\right\rangle \text { for all } i, j \\ \left(\left\langle c_{i j}, c_{i j}^{\prime}\right\rangle\right) & \text { if }\left\langle a_{i j}, a_{i j}^{\prime}\right\rangle<\left\langle c_{i j}, c_{i j}^{\prime}\right\rangle\end{cases}$

$((A \neg B) \vee(B \neg C)) \rightarrow(A \neg C)=((A \neg B) \vee(\langle 0,1\rangle)) \rightarrow(A \neg C)=$ $(A \neg B) \rightarrow(A \neg C)=(\langle 0,1\rangle)($ in all the cases $)$

Case (iii): If $C<A \leq B$

$A \rightarrow C=(\langle 0,1\rangle), B \rightarrow C=(\langle 0,1\rangle)$ and $A \rightarrow B$ is as in the previous case.

$((A \neg B) \vee(B \neg C)) \rightarrow(A \neg C)=(A \neg B) \rightarrow(\langle 0,1\rangle)=(\langle 0,1\rangle)$

5. Case (i): $A \leq B$

$B \neg((A \vee B) \neg B)=B \neg(B \neg B)=B \neg(\langle 0,1\rangle)=(\langle 0,1\rangle)$ 
Case (ii): If $A>B$

$B \rightarrow((A \vee B) \rightarrow B)=B \rightarrow(A \neg B)=B \rightarrow(\langle 0,1\rangle)=(\langle 0,1\rangle)$

6. Case (i): If $A \leq B<C$

$A \rightarrow C=C, B \rightarrow C=C,(A \wedge B) \rightarrow C=C$

$((B \rightarrow C) \rightarrow((A \wedge B) \rightarrow C)) \rightarrow(A \rightarrow C)=(C \rightarrow C) \rightarrow C=(\langle 0,1\rangle)$

Case (ii):If $A \leq C \leq B$

$B \rightarrow C=(\langle 0,1\rangle)$

$((B \neg C) \rightarrow((A \wedge B) \rightarrow C)) \rightarrow(A \rightarrow C)=((\langle 0,1\rangle) \rightarrow(A \rightarrow C)) \rightarrow$ $(A \rightarrow C)=(A \rightarrow C) \rightarrow(A \rightarrow C)=(\langle 0,1\rangle)$

Case (iii): If $C<A \leq B$

$B \rightarrow C=(\langle 0,1\rangle), A \rightarrow C=(\langle 0,1\rangle)$

$((B \neg C) \rightarrow((A \wedge B) \rightarrow C)) \rightarrow(A \rightarrow C)=((\langle 0,1\rangle) \rightarrow(\langle 0,1\rangle)) \rightarrow$ $(\langle 0,1\rangle)=(\langle 0,1\rangle)$.

\section{References}

[1] Amar Kumar Adak, Monoanjan Bhowmik and Madhumangal Pal., Some properties of Generalized Intuitionistic Fuzzy Nilpotent Matrices over Distributive Lattice, Fuzzy Inf.Engineing, Vol. 4(2012),371-387.

[2] K. Atanassov K, Intuitionistic Fuzzy Sets, VII ITKR's Session, Sofia, June 1983.

[3] K. Atanassov, Intuitionistic Fuzzy Sets ; Theory and Applications, Physica Verlag, 1999.

[4] K. Atanassov, Intuitionistic Fuzzy Implications and Modus Ponens, Notes on Intuitionistic Fuzzy Sets, Vol.(11)(1),2005.1-5.

[5] K. Atanassov, On Some Types of Fuzzy Negations, Notes on Intuitionistic Fuzzy Sets, Vol.(11)(4),2005,170-172.

[6] K. Atanassov, A New Intuitionistic Fuzzy Implication from a Modal Type, Advance Studies in Contemporary Mathematics Vol.(12)(1), 2006,117-122.

[7] K. Atanassov and G. Gargov, Elements of Intuitionistic Fuzzy Logic.Part I , Fuzzy Sets and Systems, Vol.(95)(1998),39-52. 
[8] C. Conelis, Deschrijver and K.K. Kerre, Implication in Intuitionistic Fuzzy and Interval valued Fuzzy Set Theory: construction, classification and application, Int,Journal of Approximate Reasoning, Vol.35(2004),55-95.

[9] C. Conelis, Deschrijver and K.K. Kerre, The Compositional Rule of Inference in an Intuitionistic Fuzzy Logic setting, Proceedings of the sixth ESSLLI Students Session, Kristina Striegnitz(editor),2001.

[10] J. Fodor and M. Roubens, Fuzzy Preference Modelling and Multicriteria Decision Support, Theory and Decision Library (Kulwar Academic Publishers), 1994.

[11] Hiroshi Hashimoto., Traces of Fuzzy Relations Under Dual Operations, Journal of Advanced Computational Intelligence and Intelligent Informatics, Vol. 9 (5) (2005), 563-569.

[12] Hiroshi Hashimoto., Sub-inverses of Fuzzy Matrices, Fuzzy Sets and Systems, Vol. 12 (1984), 155-168.

[13] Im Young Bin, Eun Pyo Lee and Se Won Park ., The Determinant of square Intuitionistic Fuzzy Matrices, Far East Journal of Mathematical Sciences, Vol. 3 (5) (2001), 789-796.

[14] S.K. Khan S.K, M. Pal and Amiya K Shyamal,Intuitionistic Fuzzy Matrices, Notes on Intuitionistic Fuzzy Sets, Vol. 8 (2) (2002), 51-62.

[15] A.R. Meenakshi, and T. Gandhimathi, Intuitionistic Fuzzy Relational Equations, advances in Fuzzy Mathematics, Vol. 5 (3) (2010), 239-244.

[16] P. Murugadas and K. Lalitha, Dual implication Operator in Intuitionistic Fuzzy Matrices, Int.Conference on Mathematical Modelling and its Applications-Dec 22-24,2012, Organized by Department of Mathematics, Annamalai University.

[17] P. Murugadas and K. Laitha,Bi-implication Operator on Intuitionistic Fuzzy Set, Journal of advances in Mathematics, Vol. 6(2), (2014), 961-969.

[18] P. Murugadas and K. Lalitha,Sub-inverse and g-inverse of an Intuitionistic Fuzzy Matrix Using Bi-implication Operator, Int.Journal of Computer Application, Vol. 89(1), (2014), 1-5.

[19] P. Murugadas and K. Lalitha,Implication Operator on Intuitionistic Fuzzy Tautological Matrix, Int.Journal of Fuzzy Mathematical Archive, Vol. 5(2), (2014), 79-87. 
[20] S. Sriram and P. Murugadas, Sub-inverses of Intuitionistic Fuzzy Matrices, Acta Ciencia Indica(Mathematics), Vol.XXXVII, M N0.1(2011), 41-56.

[21] S. Sriram and P. Murugadas, Contribution to a study on Generalized Fuzzy Matrices, Ph.D Thesis Department of Mathematics, Annamalai University, July-2011.

[22] S. Sriram and P. Murugadas., On Semi-ring of Intuitionistic Fuzzy Matrices, Applied Mathematical Science, Vol. 4(23), (2010), 1099-1105.

[23] Z. Xu and R.R. Yager,Some geometric operators based on Intuitionistic Fuzzy Sets, Int. Journal of General Systems, Vol. 35(2006), 417-433.

[24] L.A. Zadeh, Fuzzy Sets,Journal of Information and Control, Vol.8(1965), 338-353. 